Supporting Information

\title{
Enhanced Antimalarial Efficacy Obtained by Targeted Delivery of Artemisinin in Heparin-Coated Magnetic Hollow Mesoporous Nanoparticles
}

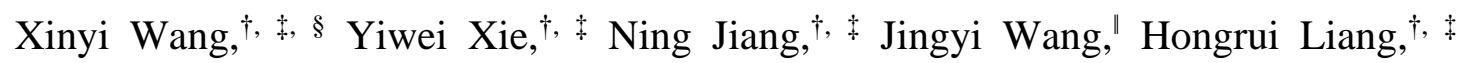

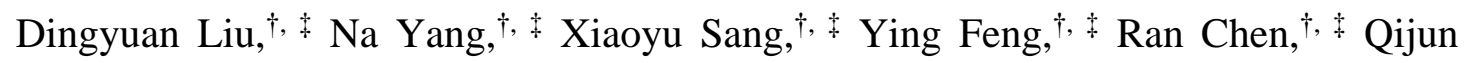
Chen, $*, \dagger, *$

$\dagger$ Key Laboratory of Livestock Infectious Diseases in Northeast China, Ministry of Education, Key Laboratory of Zoonosis, College of Animal Science and Veterinary Medicine, Shenyang Agricultural University, 120 Dongling Road, Shenyang 110866, China.

$\ddagger$ The Research Unit for Pathogenic Mechanisms of Zoonotic Parasites, Chinese Academy of Medical Sciences, 120 Dongling Road, Shenyang 110866, China.

$\S$ College of Basic Sciences, Shenyang Agricultural University, 120 Dongling Road, Shenyang 110866, China.

॥ College of Information and Electrical Engineering, Shenyang Agricultural University, 120 Dongling Road, Shenyang 110866, China. 


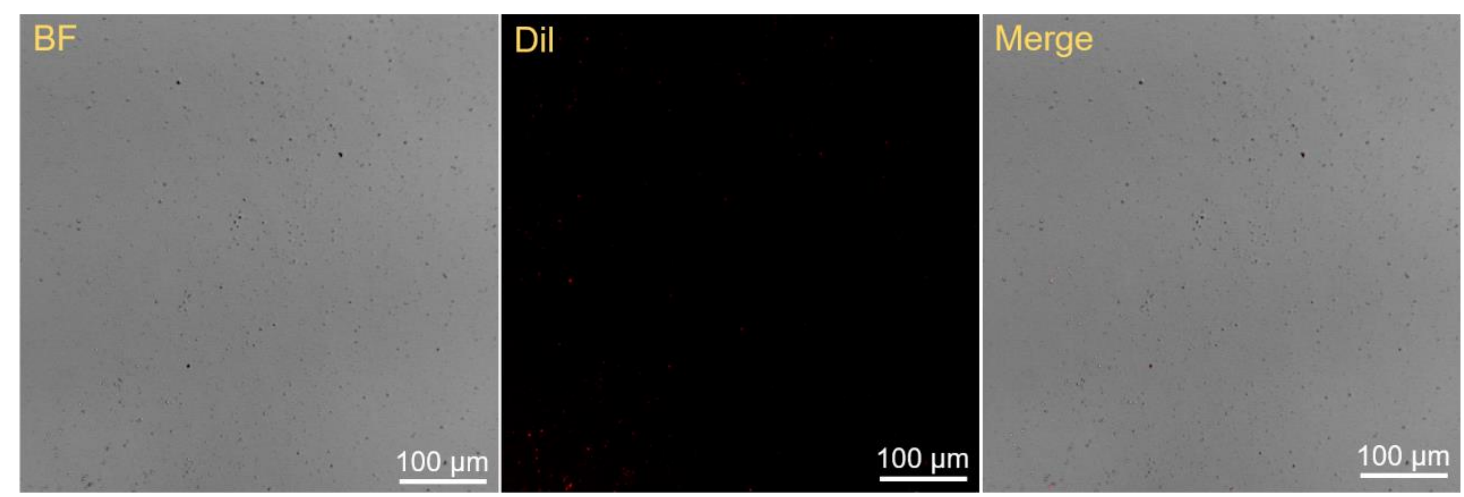

Figure S1. The dispersity of HMFNs in the complete medium containing 10\% FBS by fluorescent imaging. HMFNs was labeled with fluorescence dye of Dil (HMFNs@Dil). 

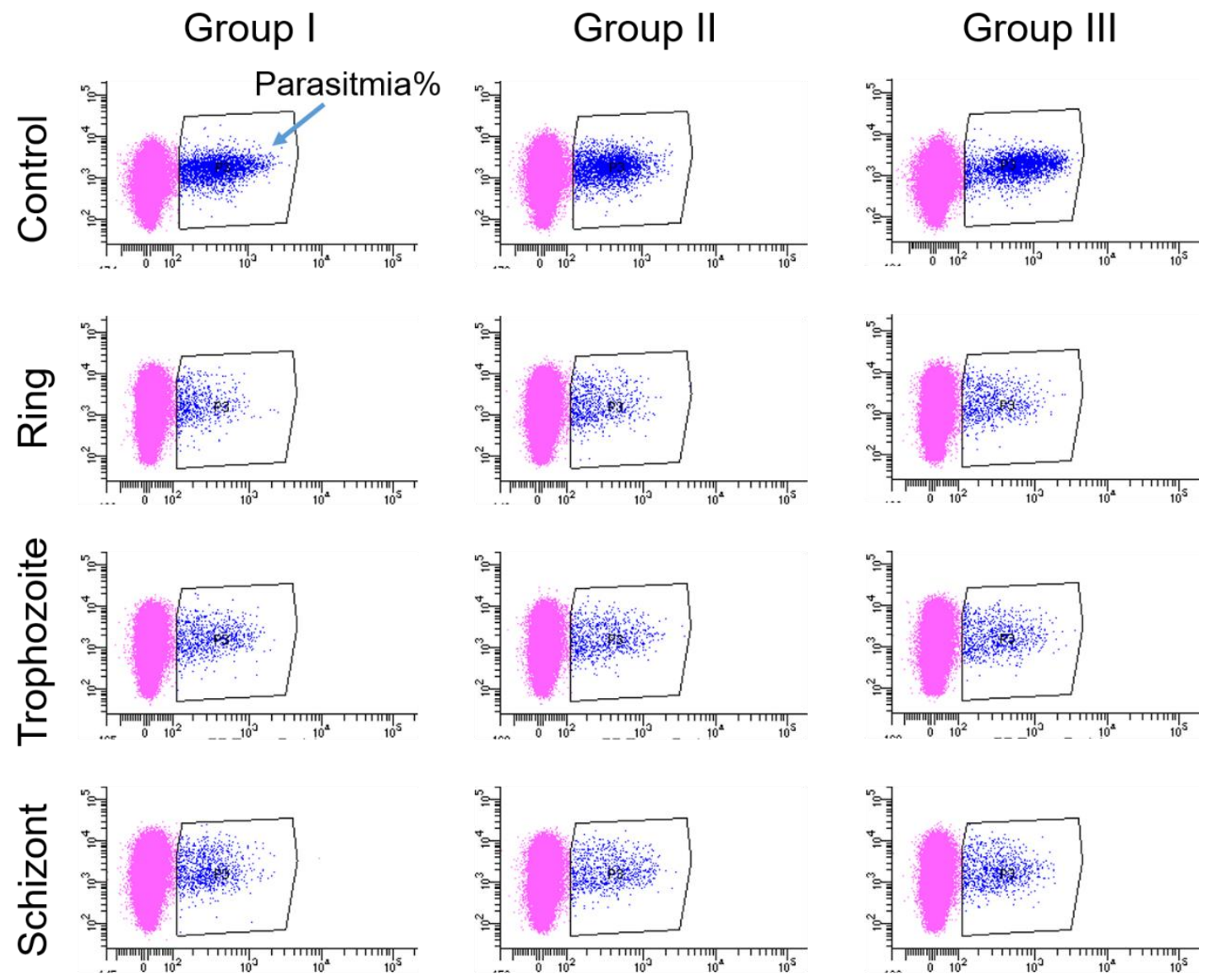

Figure S2. Flow cytometry analysis of parasitemia (three independent experiments) by staining the nucleus of $P$. falciparum with DHE. HMFNs $(200 \mu \mathrm{g} / \mathrm{mL})$ were added at different developmental stages of $P$. falciparum involving Ring, Trophozoite, and Schizont, and the parasitemia were determined at the ring phase of the next cycle. The iRBC cells without treatment were used as control. 

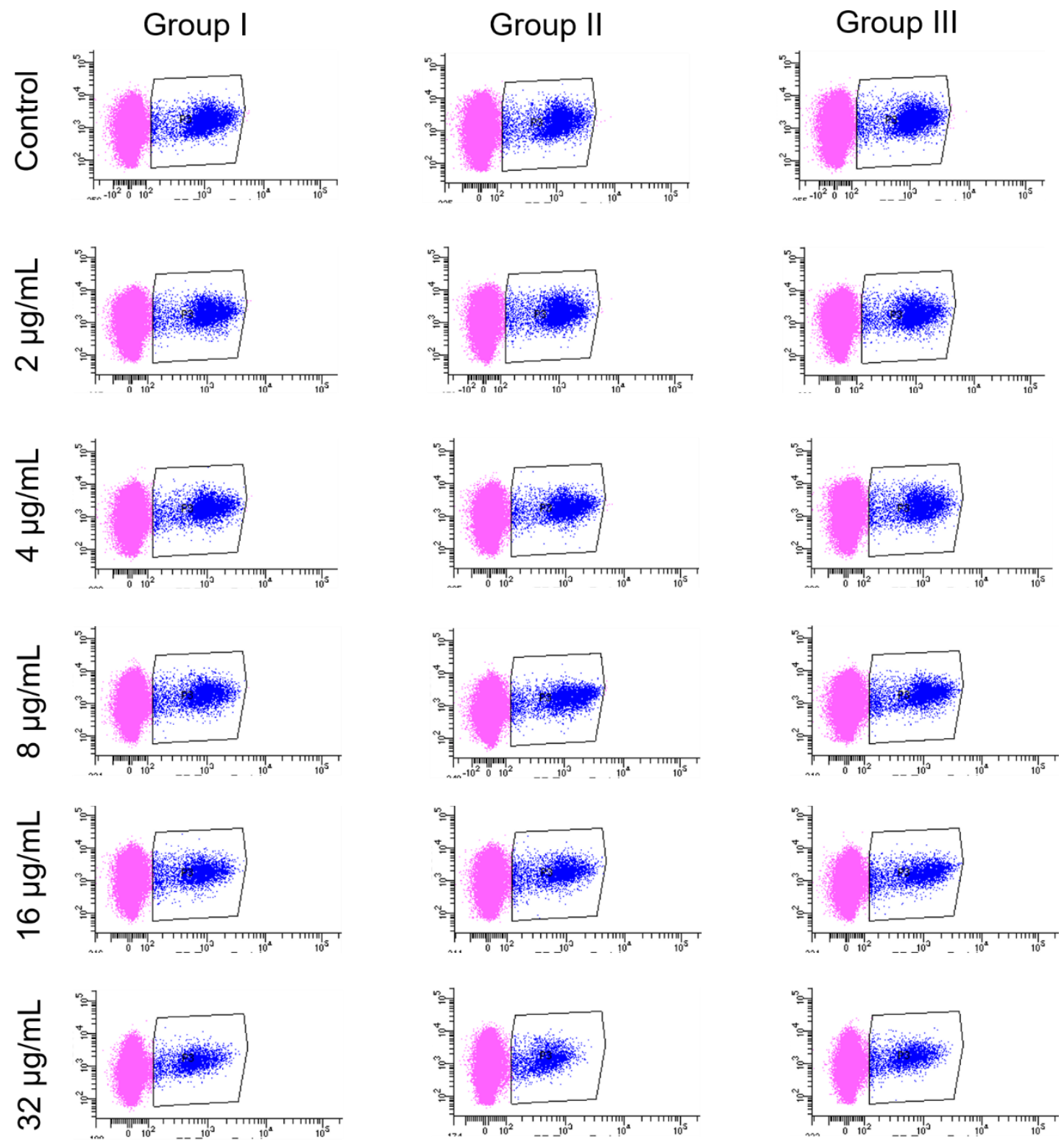

Figure S3. Flow cytometry analysis of parasitemia (three independent experiments) after the treatment of heparin with different concentrations by staining the nucleus of $P$. falciparum with DHE. Heparin $(2,4,8,16$ and $32 \mu \mathrm{g} / \mathrm{mL})$ were added at schizont phase and the parasitemia were determined at the trophozoite phase of the next cycle. The iRBC cells without treatment were used as control. 
Group I
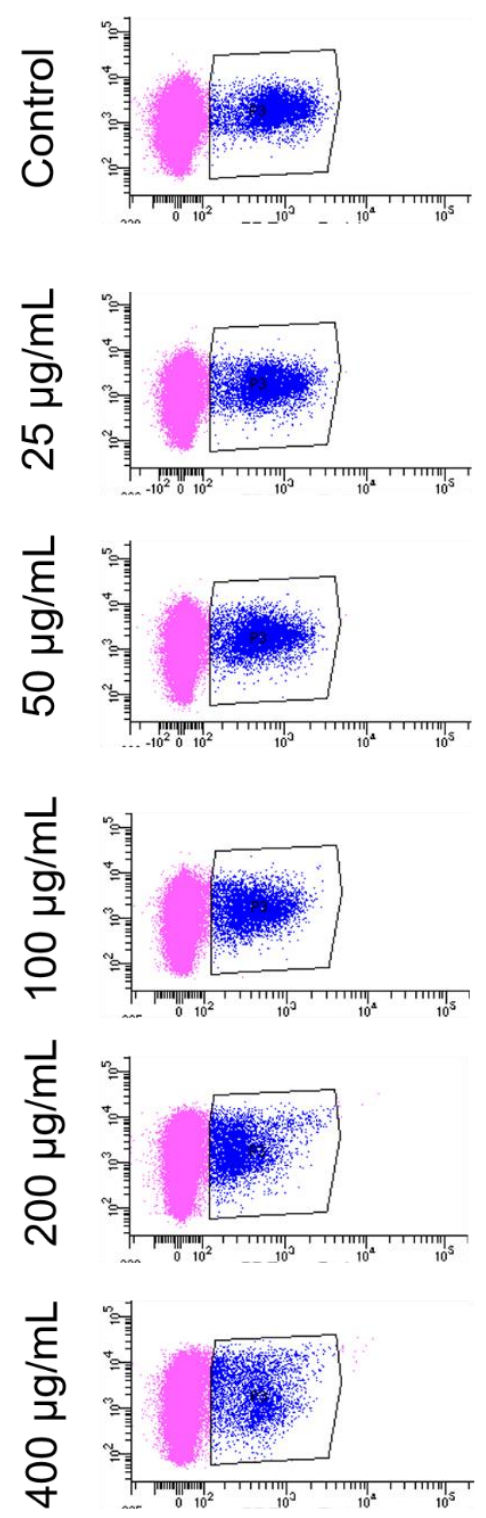

Group II
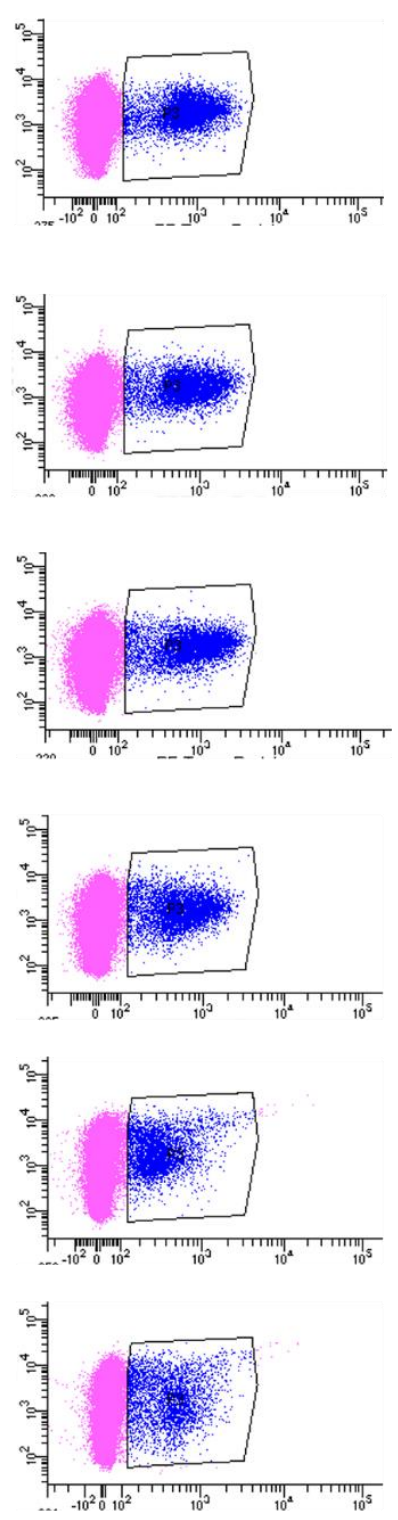

Group III
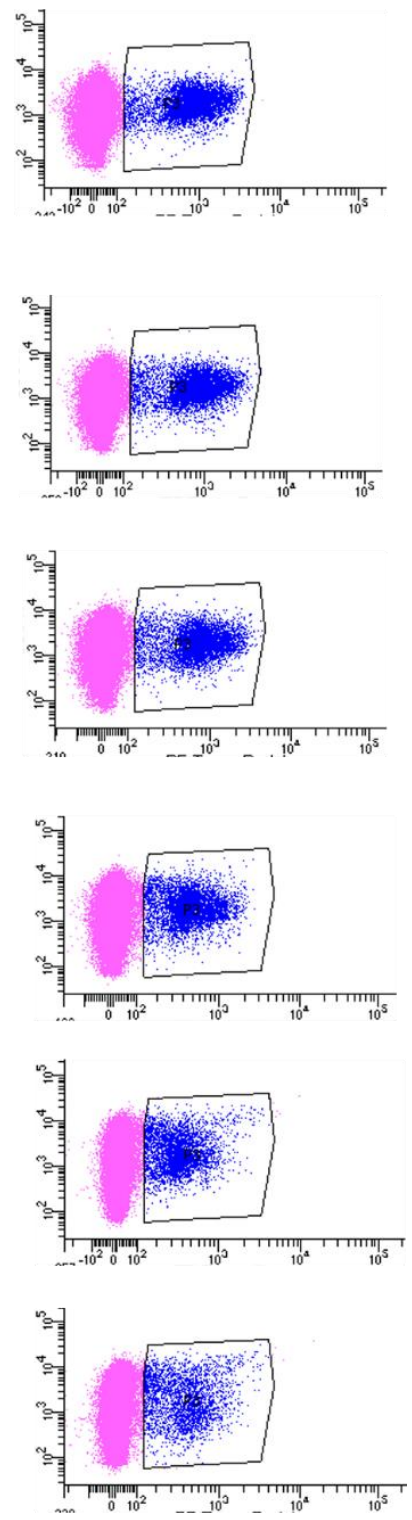

Figure S4. Flow cytometry analysis of parasitemia (three independent experiments) after the treatment of HMFNs with different concentrations by staining the nucleus of P. falciparum with DHE. HMFNs $(25,50,100,200$ and $400 \mu \mathrm{g} / \mathrm{mL})$ were added at schizont phase and the parasitemia were determined at the trophozoite phase of the next cycle. The iRBC cells without treatment were used as control. 


\section{Group I}
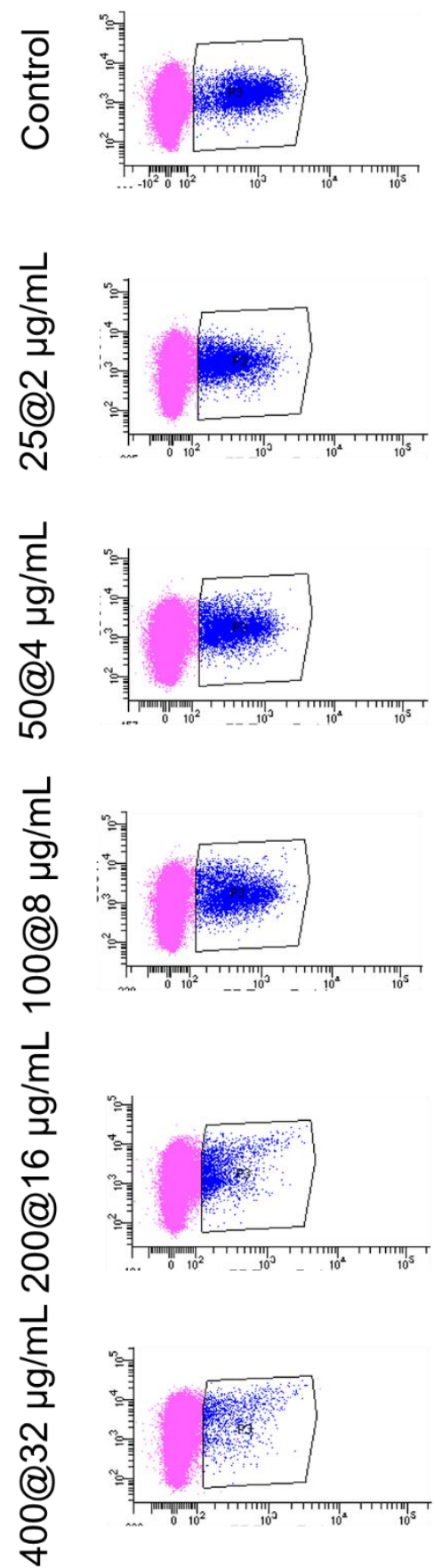

Group II
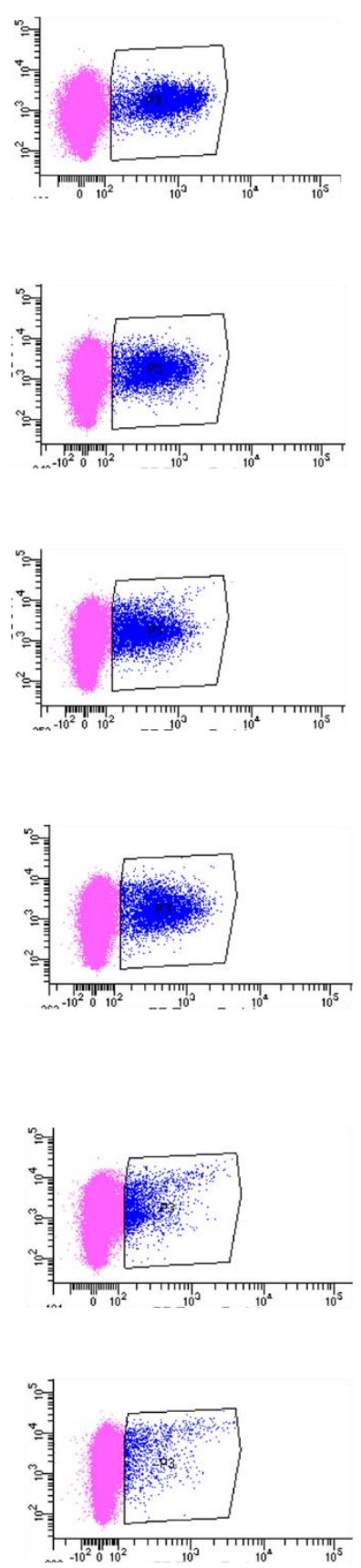

Group III
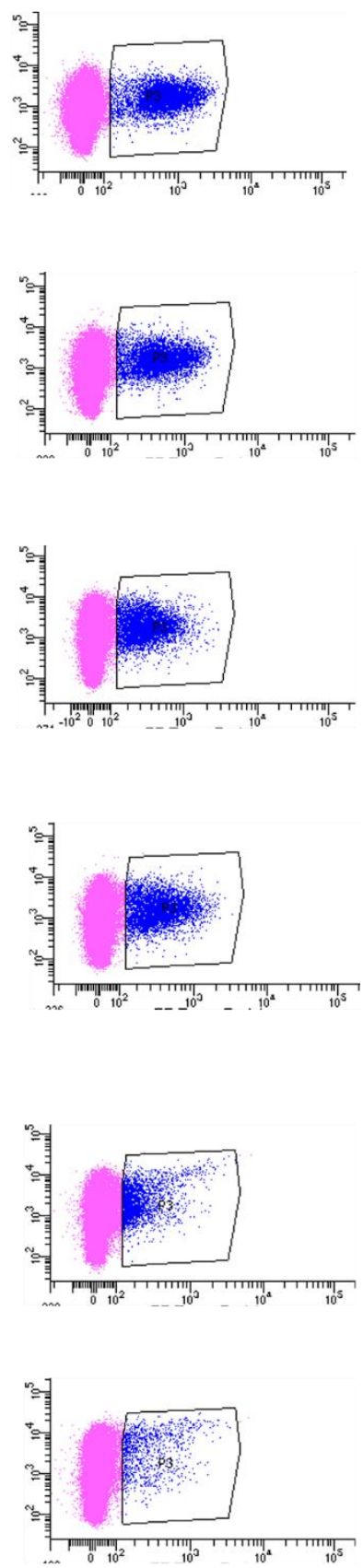

Figure S5. Flow cytometry analysis of parasitemia (three independent experiments) after the treatment of HMFN@HEP with different concentrations by staining the nucleus of P. falciparum with DHE. HMFN@HEP (25@2, 50@4, 100@8, 200@16 and 400@32 $\mu \mathrm{g} / \mathrm{mL}$ ) were added at schizont phase and the parasitemia were determined at the trophozoite phase of the next cycle. The iRBC cells without treatment were used as control. 


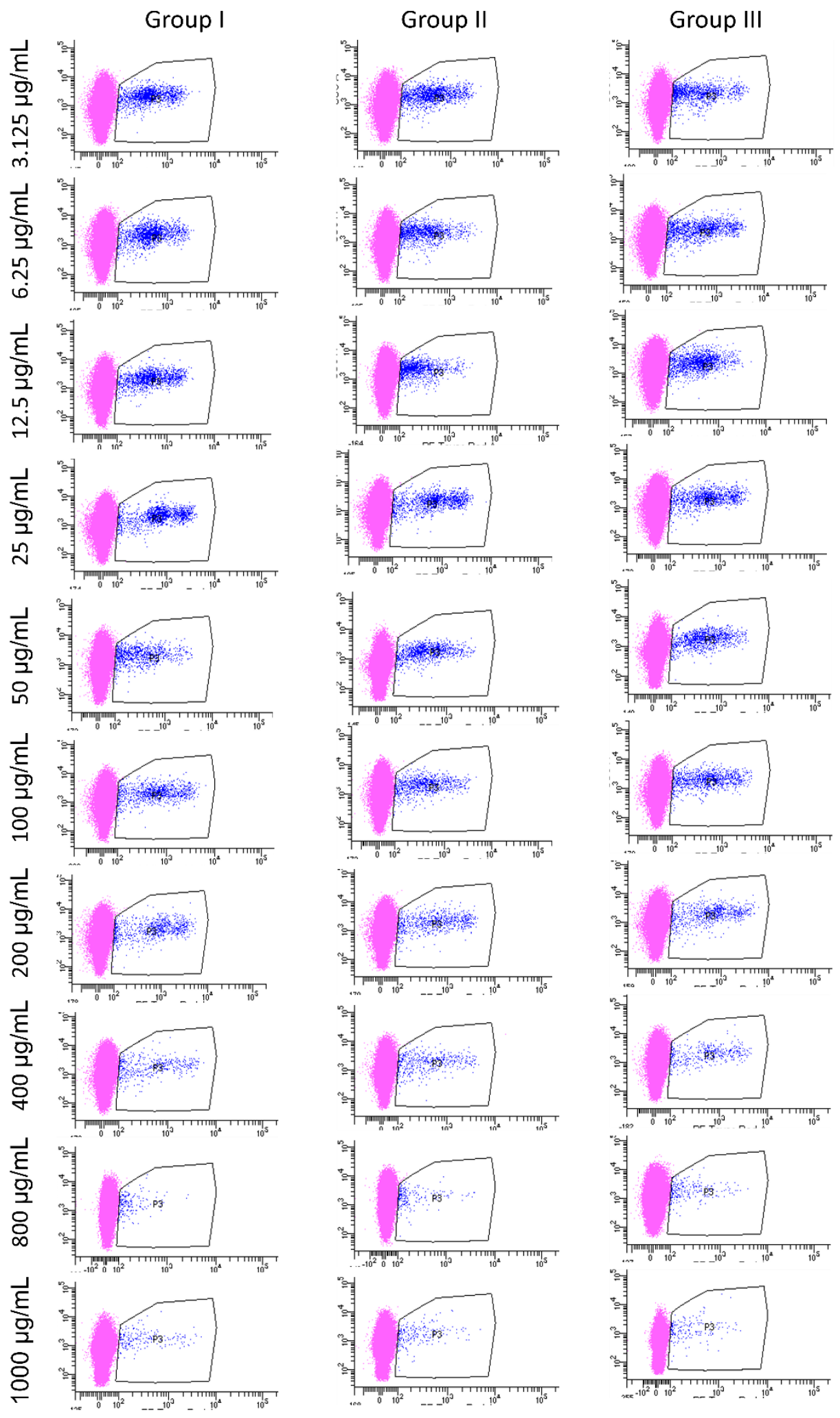

Figure S6. Flow cytometry analysis of parasitemia (three independent experiments) after the treatment of free heparin with various concentrations. All data points were the percentage of control treated by PBS. The iRBC cells were treated by free heparin for $72 \mathrm{~h}$. 

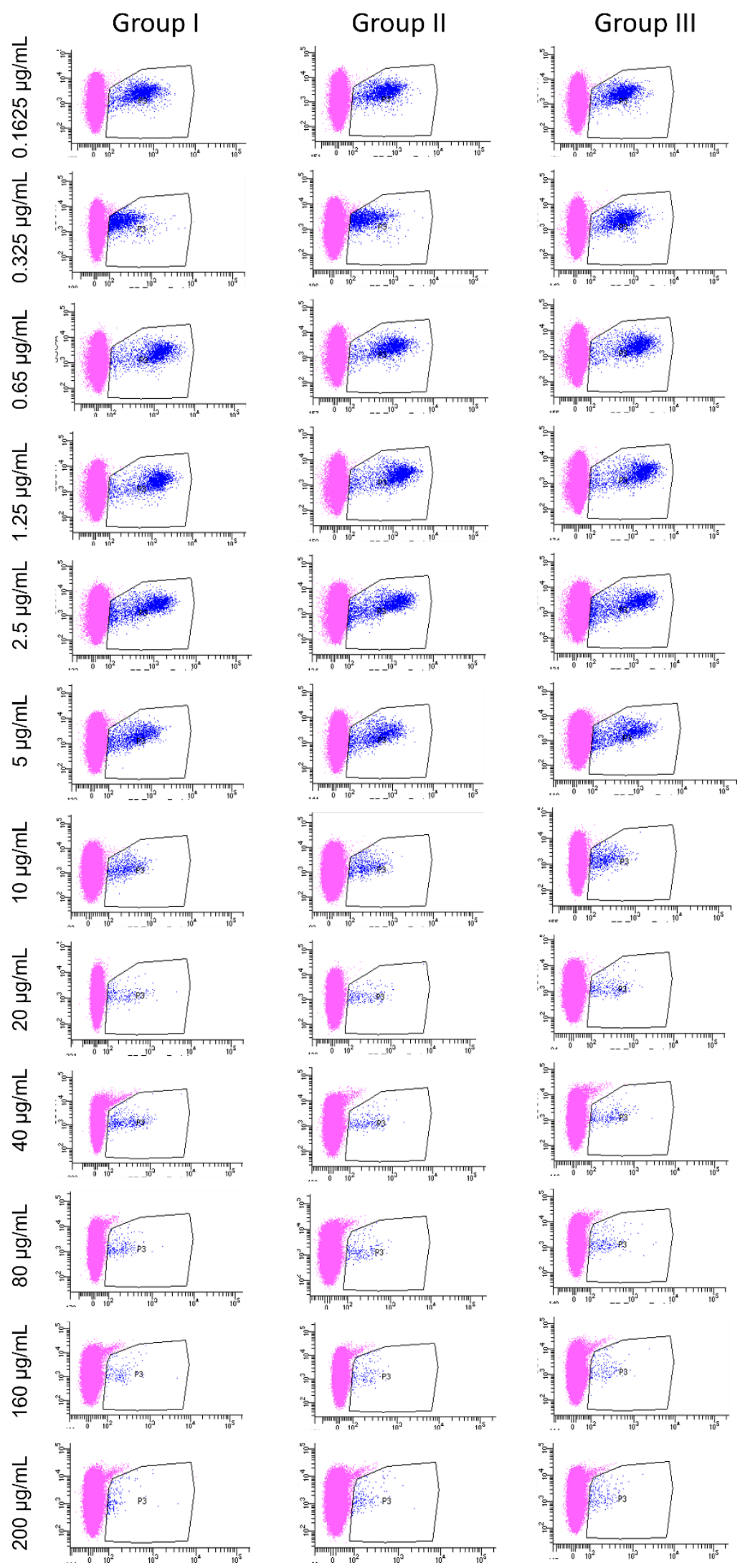

Figure S7. Flow cytometry analysis of parasitemia (three independent experiments) after the treatment by various concentrations of HMFN@HEP (5.3\% HEP loading efficiency). All data points were the percentage of control treated by PBS. The iRBC cells were treated by HMFN@HEP for 72h. 

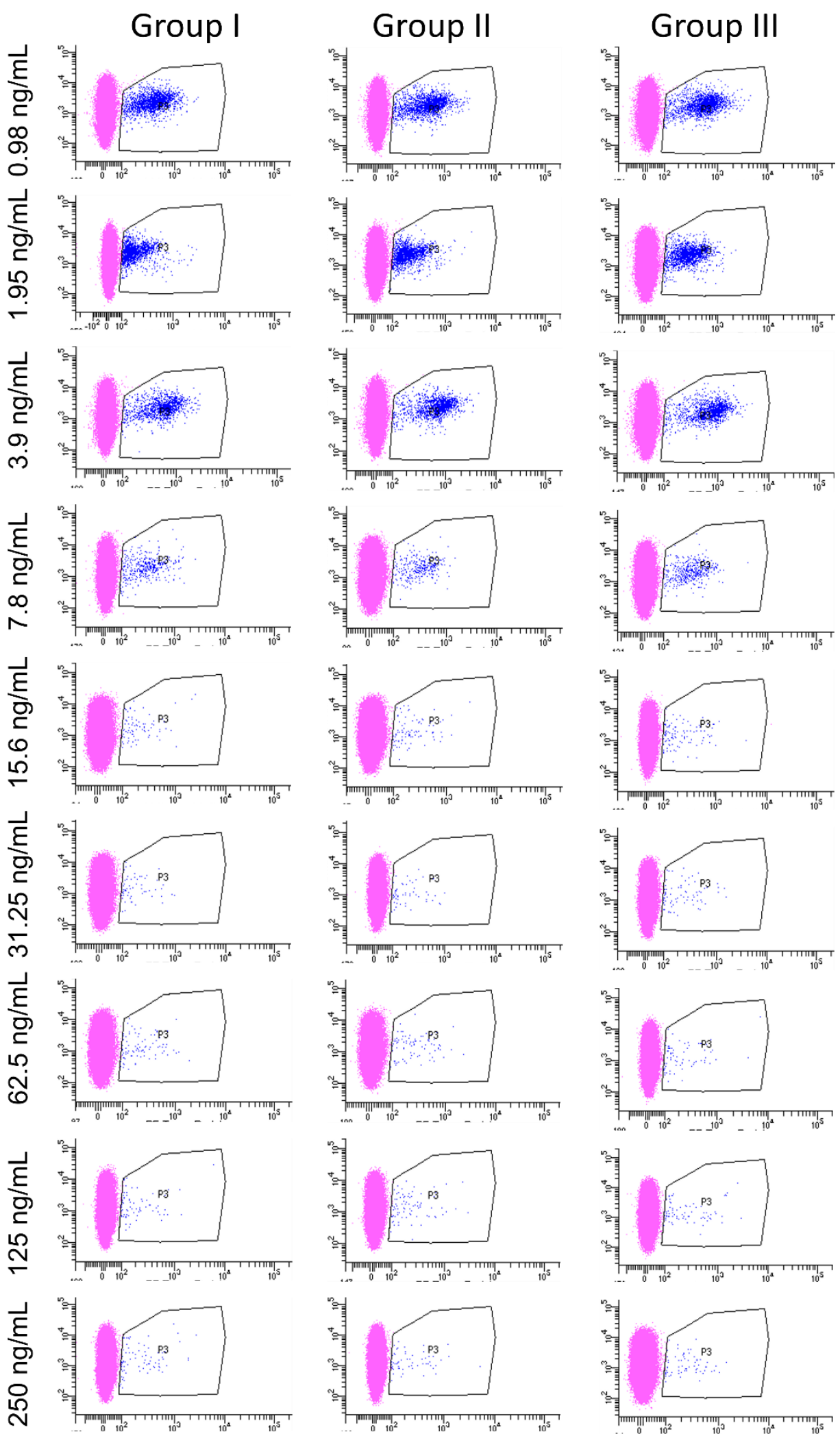

Figure S8. Flow cytometry analysis of parasitemia (three independent experiments) after the treatment by various concentrations of HMFN@ART@HEP (6.5 wt\% of ART and $5.3 \mathrm{wt} \%$ of HEP loading efficiency). All data points were the percentage of control treated by PBS. The iRBC cells were treated by HMFN@HEP for 72h. 
Group I
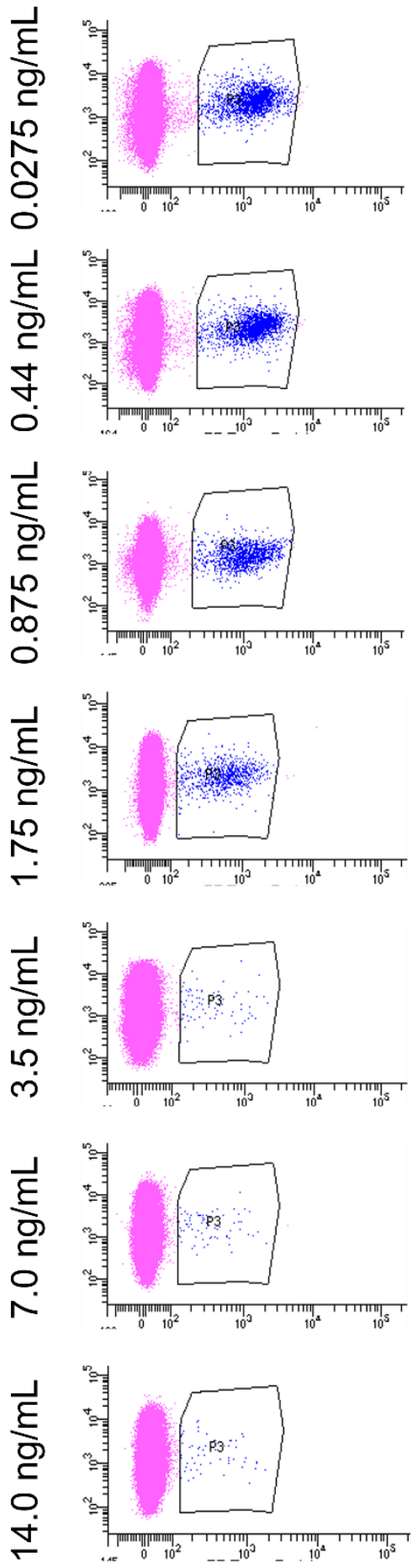

Group II
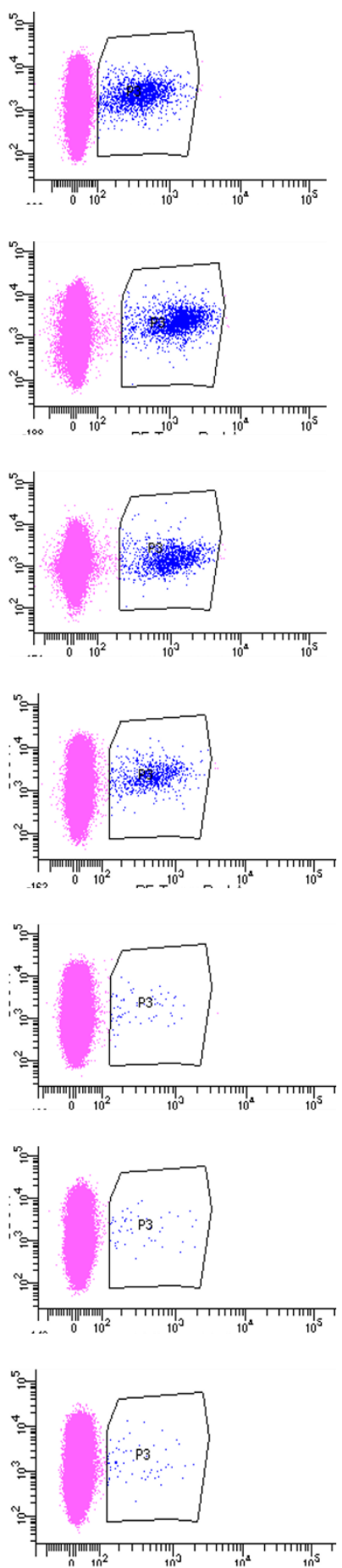

Group III
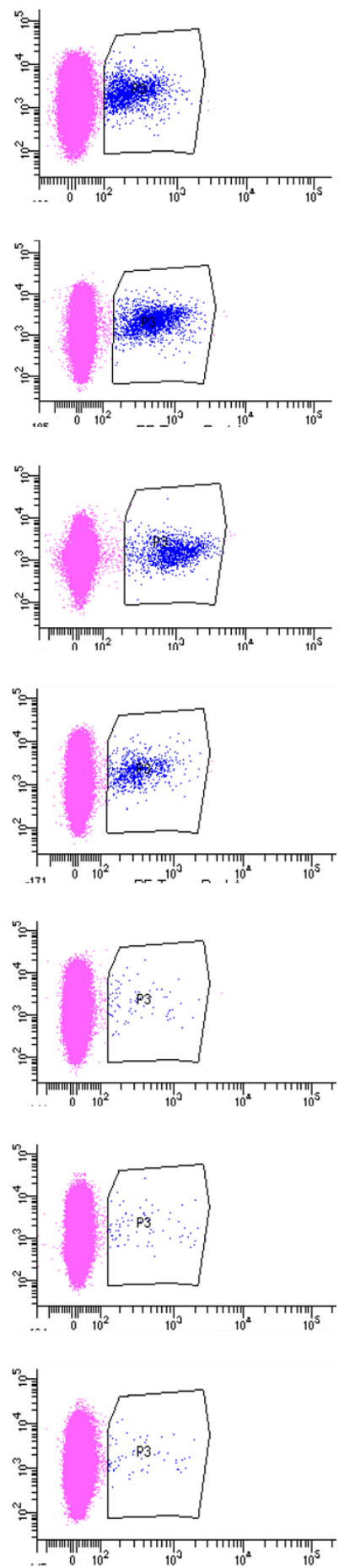

Figure S9. Flow cytometry analysis of parasitemia (three independent experiments) after the treatment of free artemisinin with various concentrations. All data points were the percentage of control treated by PBS. The iRBC cells were treated by free artemisinin for $72 \mathrm{~h}$. 

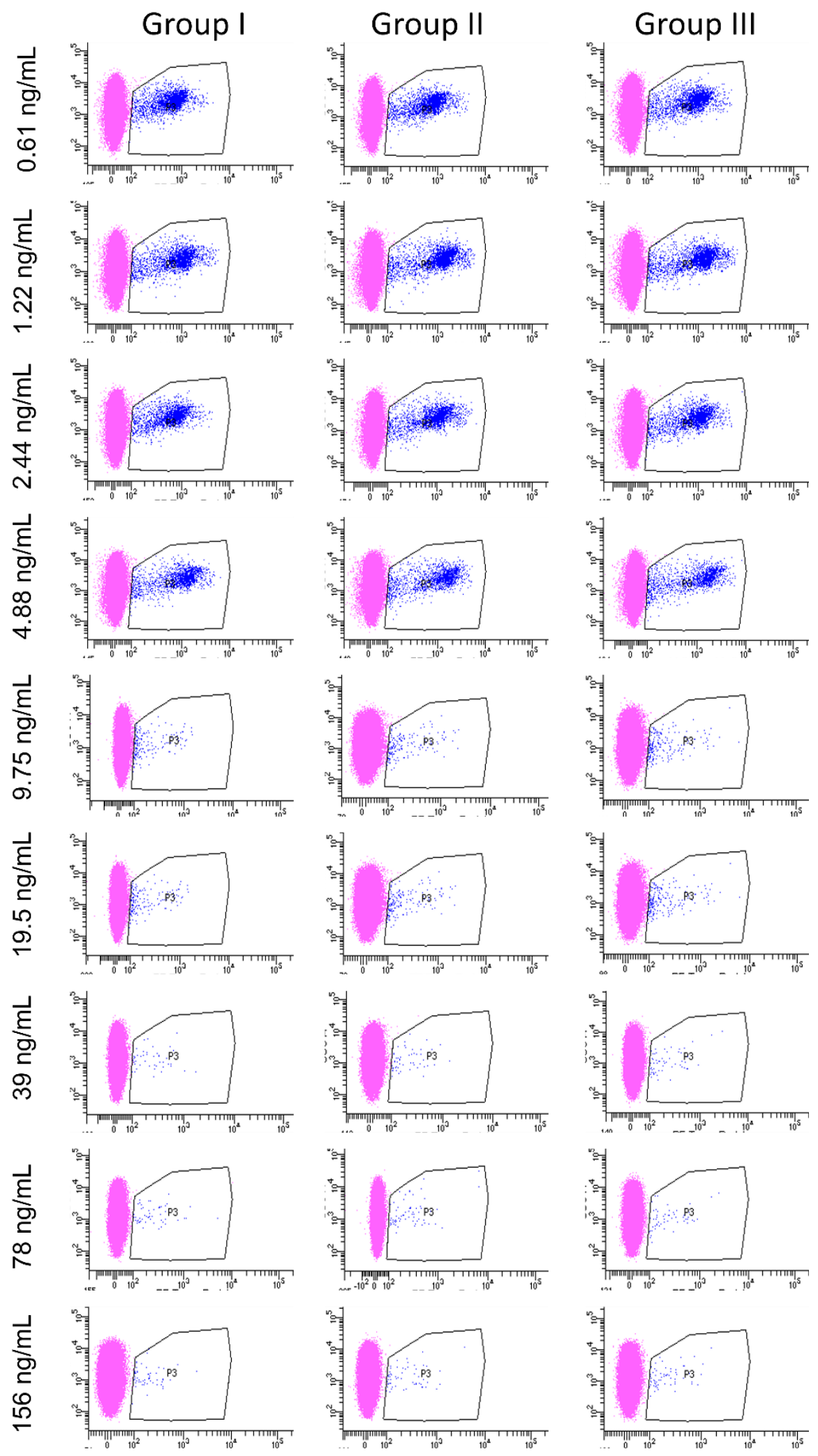

Figure S10. Flow cytometry analysis of parasitemia (three independent experiments) after the treatment with various concentrations of HMFN@ART (6.5\% ART loading efficiency). All data points were the percentage of control treated by PBS. The iRBC cells were treated by HMFN@ART for 72h. 

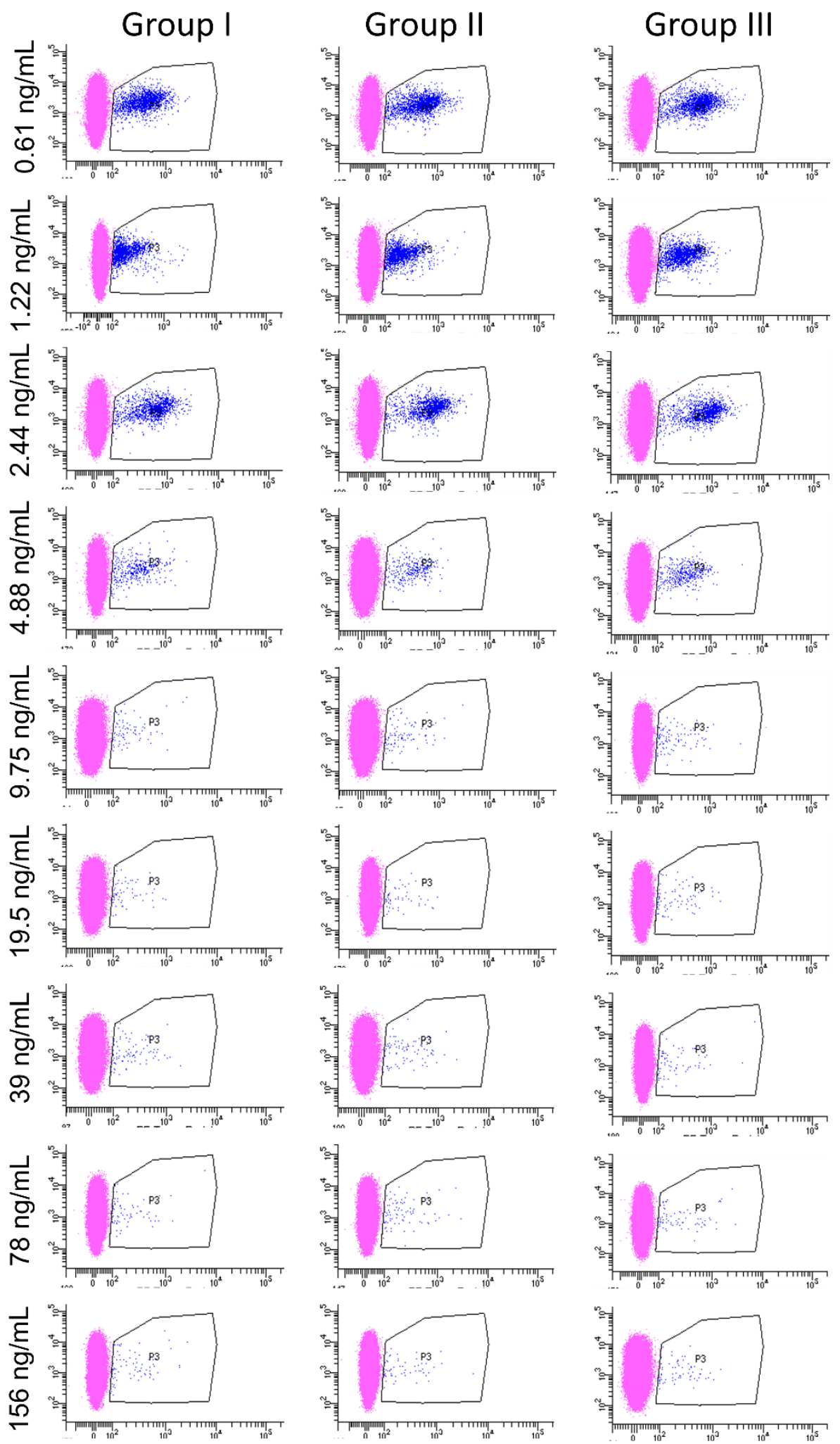

Figure S11. Flow cytometry analysis of parasitemia (three independent experiments) after the treatment by various concentrations of HMFN@ART@HEP (6.5 wt\% of ART and $5.3 \mathrm{wt} \%$ of HEP loading efficiency). All data points were the percentage of control treated by PBS. The iRBC cells were treated by HMFN@HEP for 72h. 

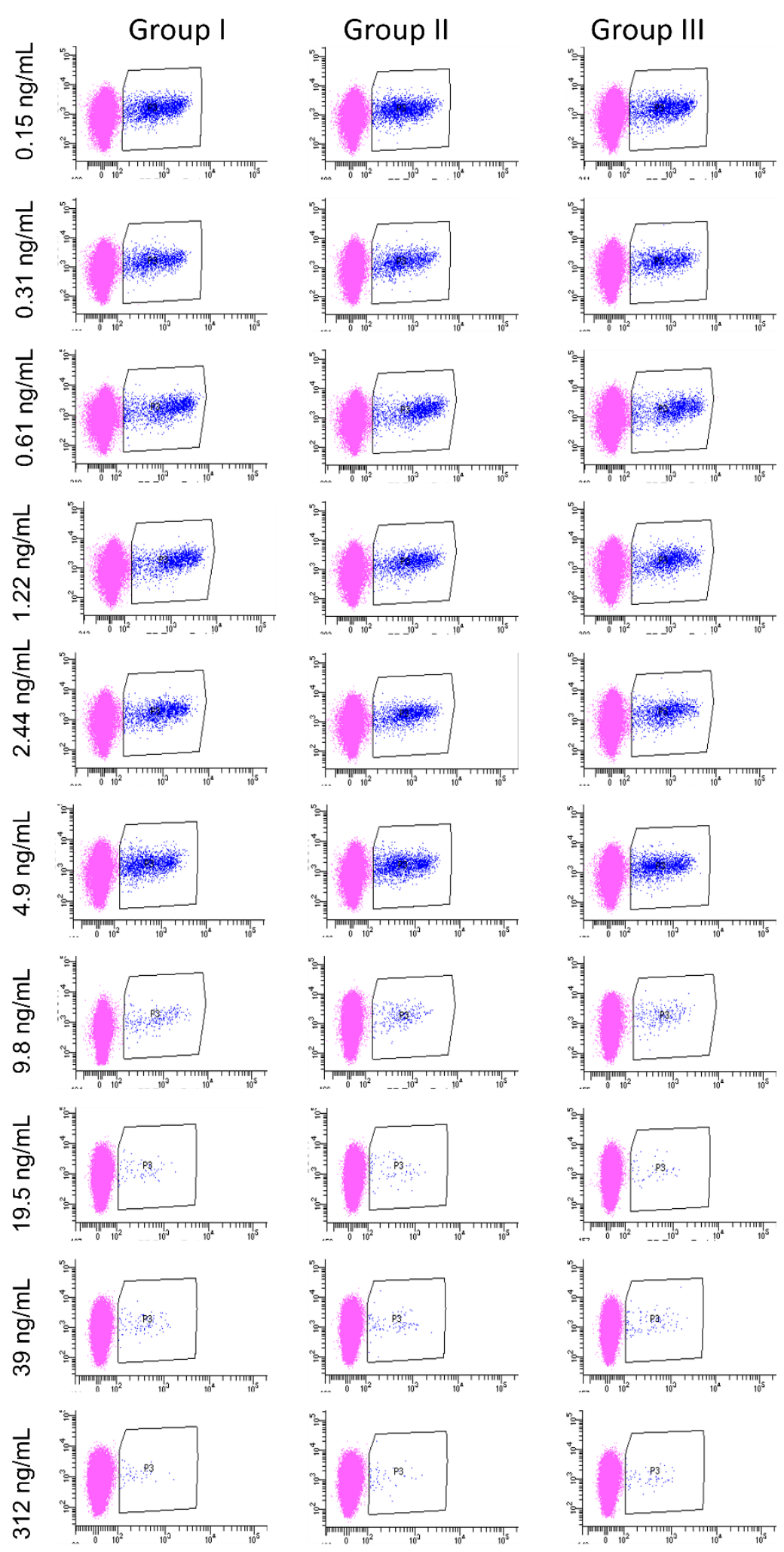

Figure S12. Flow cytometry analysis of parasitemia (three independent experiments) after the treatment with HMFN@ART@HEP loading 5.3\% HEP and various concentrations of ART. The concentration of HMFN nanocarriers was $50 \mu \mathrm{g} / \mathrm{mL}$. All data points were the percentage of control treated by PBS. The iRBC cells were treated for $72 \mathrm{~h}$. 

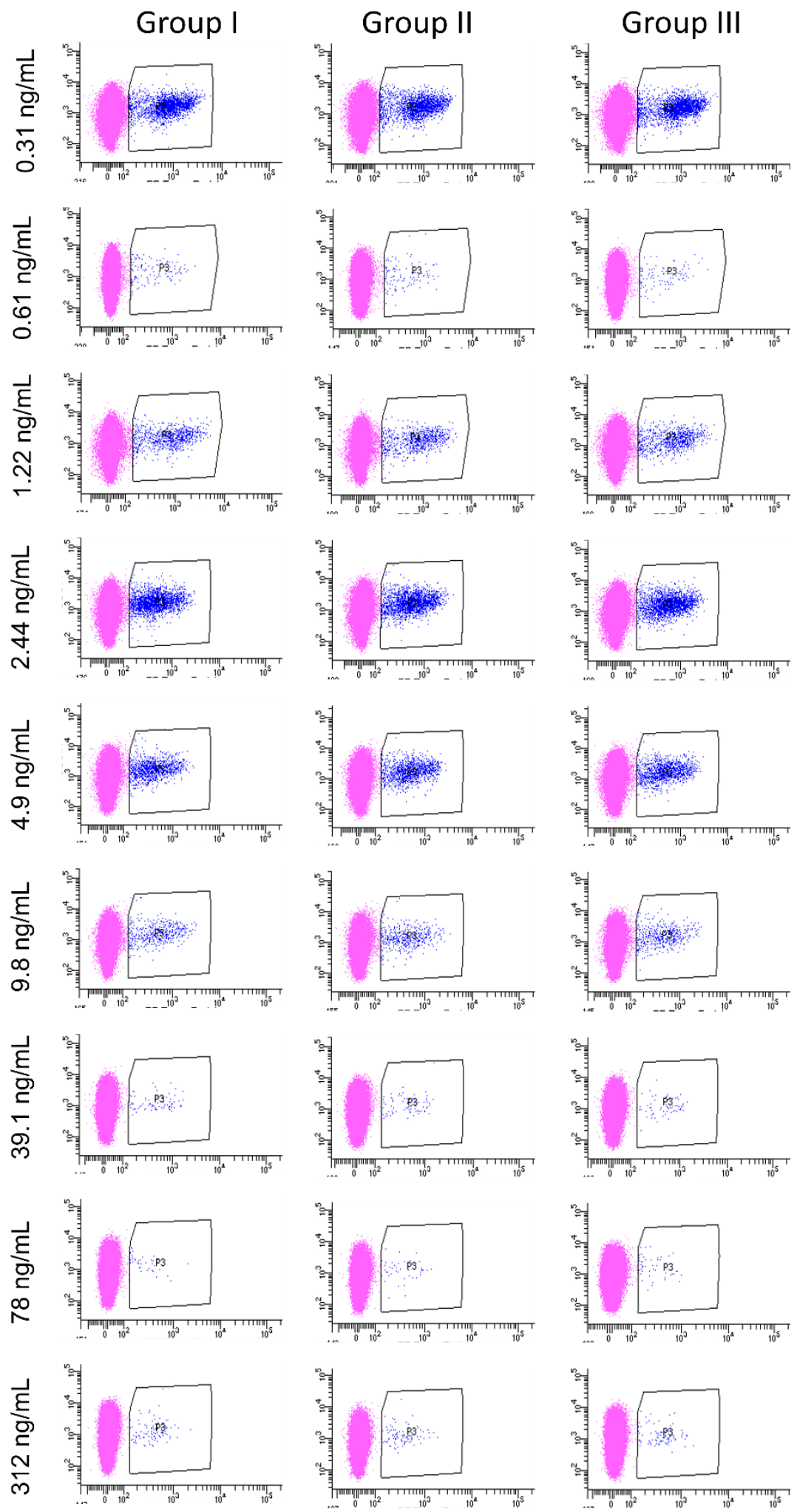

Figure S13. Flow cytometry analysis of parasitemia (three independent experiments) after the treatment with HMFN@ART@HEP loading 5.3\% HEP and various concentrations of ART. The concentration of HMFN nanocarriers was $100 \mu \mathrm{g} / \mathrm{mL}$. All data points were the percentage of control treated by PBS. The iRBC cells were treated for $72 \mathrm{~h}$. 

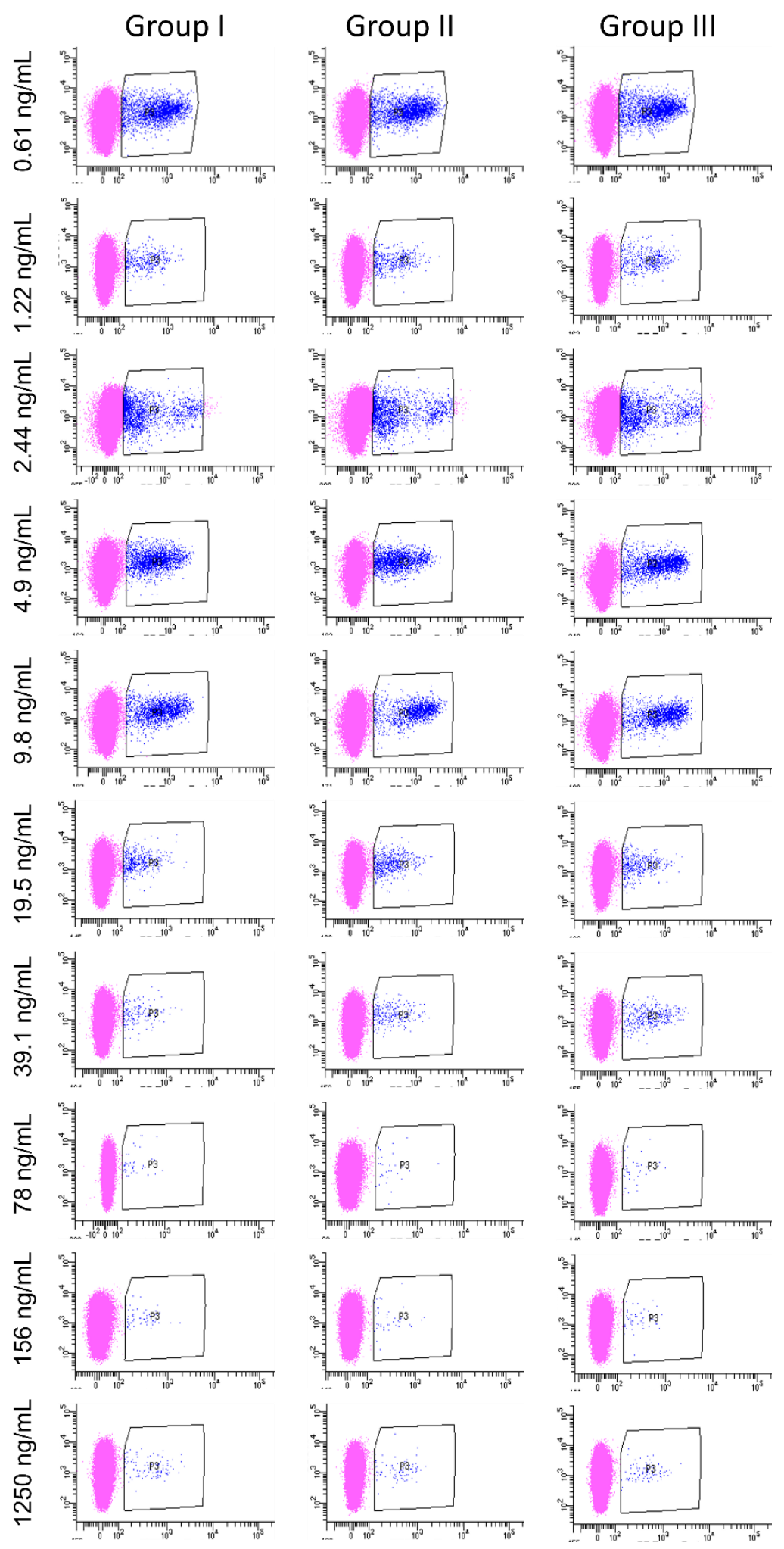

Figure S14. Flow cytometry analysis of parasitemia (three independent experiments) after the treatment with HMFN@ART@HEP loading 5.3\% HEP and various concentrations of ART. The concentration of HMFN nanocarriers was $200 \mu \mathrm{g} / \mathrm{mL}$. All data points were the percentage of control treated by PBS. The iRBC cells were treated for $72 \mathrm{~h}$. 